\title{
The Discovery of the First Exoplanet Orbiting a Solar-Type Star
}

\author{
Didier Queloz, Mejd Alsari`
}

\begin{abstract}
Didier Queloz is Professor of Physics at the Cavendish Laboratory (University of Cambridge) and Geneva University. He was jointly awarded the $\mathbf{2 0 1 9}$ Nobel Prize in Physics for "the discovery of an exoplanet orbiting a solar-type star". In the first part of his conversation with Mejd Alsari he discusses the impact of his 1995 discovery on the theory of planetary systems formation. See video at https://youtu.be/6xqbBWDgzsY
\end{abstract}

Mejd Alsari (MA). In 1995 you dramatically changed the view we had about planet formation in the Universe with the discovery of the first giant planet outside our solar system. This discovery started a revolution in astronomy and in 2019 you shared the Nobel Prize in Physics with Michel Mayor for the discovery of an exoplanet orbiting a solar-type star. Can you summarise the key findings in the paper you published in Nature in 1995 ? $^{1}$

Didier Queloz (DQ). We identified the first planet orbiting another star other than the Sun. That was a key discovery. It was a trigger for the field because, up to that day, people were hoping that there are planets somewhere orbiting other stars, but no one had really found one. ${ }^{2-5}$

What came with the discovery was a lot of embarrassment as well because the planet wasn't at all the way we expected it to be. We found a big planet (51 Pegasi b) which was the only one we could detect. In fact, due to instrumentation limits, we could only detect relatively large planets. ${ }^{1}$

The problem of that planet was its orbit, which was extremely close to its star, about 20 times closer to its star than the orbit of the Earth to the Sun, and that was really awkward. We call these planets hot Jupiters. The theories of planetary formations were not predicting such a planet. ${ }^{6}$ In addition to the discovery of the first exoplanet orbiting a main-sequence star, we broke the theory.

That was really the main impact of this discovery almost 25 years ago.

MA. According to our former understanding of the formation of solar systems, 51 Pegasi b shouldn't be where it is now. The past 30 years of discoveries tell us that our solar system is very unusual. Where are we now in terms of planetary system models? Can you compare between models back in 1995 and now?

DQ. This is a very interesting question. The first discovery was awkward, but all the other discoveries that came later on were awkward as well because we kept detecting planets that no one had predicted. ${ }^{7-15}$ We have plenty of hot Jupiters but we also have objects we had no idea they would exist like hot Earths or super Earths ${ }^{16}$ or hot mini Neptunes ${ }^{17}$. We have this kind of population of planets that we cannot directly compare to the ones in the Solar System.

We have a very detailed theory that is working pretty well to explain the formation and nature of our own system. ${ }^{18}$ We have a lot of data on the planets of our Solar System, including remnant bodies from the early Solar System, which sometimes fall on Earth as asteroids. ${ }^{19}$ There are lots of elements that we can put together. We know the atmospheric composition of at least one giant planet in the Solar System. ${ }^{20}$ We have a good understanding of the telluric planets, maybe not Mercury, which is not very well-known.

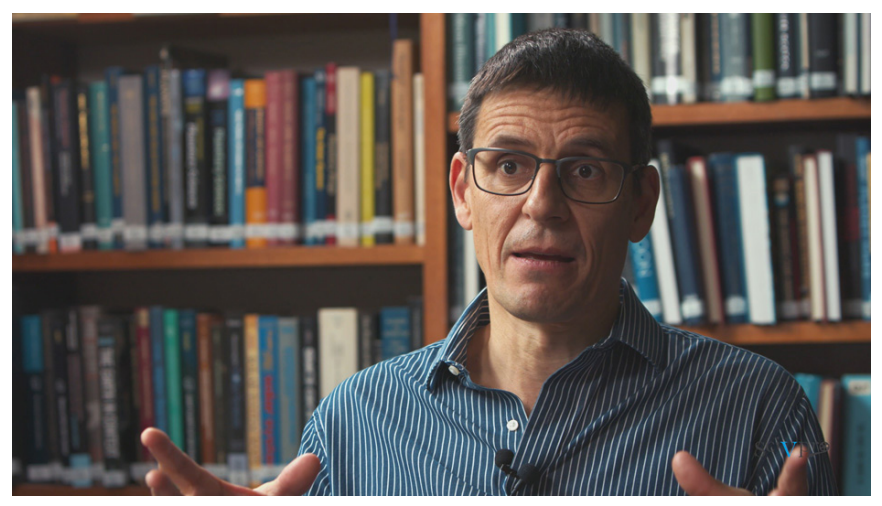

Figure 1 | Prof. Didier Queloz, Professor of Physics, Cavendish Laboratory.

I think discoveries of exoplanets don't challenge our understanding of the Solar System, but tell us that this picture is one amongst many possibilities to form planets.

We have expanded our understanding of planetary system models by adding lots of ingredients. One of them is the fact that the planet can, in a way, move during the early stage a lot more than we thought. A planet can form at a certain location and then can move towards its star. We call this migration. ${ }^{21-23}$ We can also have a multi-planetary system, where planets interact. ${ }^{24-26}$ Due to this interaction, planets can move outwards or inwards in the planetary system. In this case, it becomes difficult getting a clear understanding of how to connect the end product to the initial stage of a planetary system evolution, because so many things can happen. Right now we are trying to retrieve as much data as we can from many planets in order to go back in time to reconstruct all the steps of planetary systems evolution.

This data includes parameters such as mass and size, but also information from the atmosphere of these planets. ${ }^{27-29}$ This can tell us part of the story on the origin of the chemical constituents of a planet, whether they have been accreted by the planet or built-up in the planet.

This means that it's not enough to detect them, get the mass, and the size. We really want to know more about the atmosphere of these planets as we've been doing in the Solar System when we studied the atmosphere of the giant planets. Our models tell us that the giant planets or the ones that look big enough to have a lot of gas, may have formed in the outskirts of the Solar System due to the fact that this is where the ingredients were in a kind of solid form that could have easily accreted on the planet. ${ }^{30-32}$ This is known as 


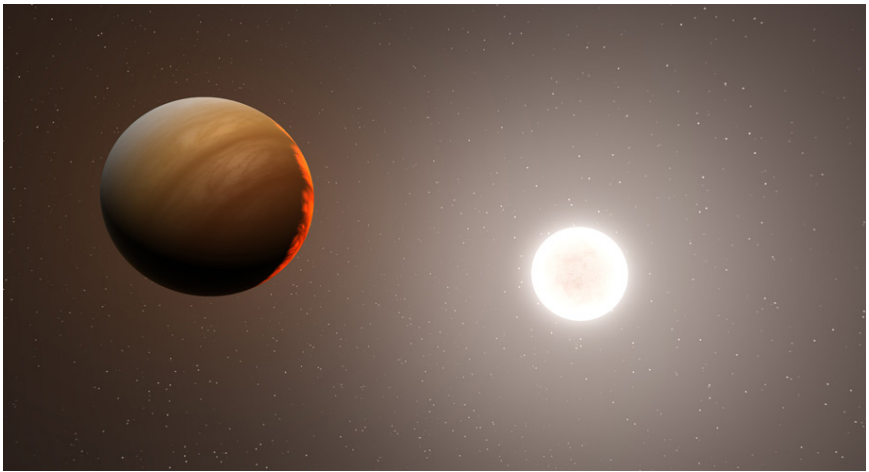

Figure 2 | Artist impression of the exoplanet 51 Pegasi b (generated with Universe Sandbox).

the formation mechanism of giant planets beyond the ice line, , $^{33-35}$ where by ice we indicate not only water but also any other gas that can solidify.

The general theory of planetary system formation is trying to connect the detailed data we have on the Solar System with the kind of loose data we have on other planets. We have not reached a complete agreement yet because we haven't found enough planets that look like the ones in the Solar System, such as Earth or Venus.

When we go back to the Solar System, we ask ourselves: 'Why the Solar System didn't move?' This is part of the key ongoing questions we are trying to solve.

There are several space missions that we hope will help addressing these questions. There is an operational space mission called TESS (Transiting Exoplanet Survey Satellite), which is trying to identify more transiting systems. ${ }^{36,37}$ Another mission that will be launched soon is CHEOPS (CHaracterising ExOPlanets Satellite mission), which will give a more detailed analysis of these transits. ${ }^{38,39}$ The James Webb Space Telescope (JWST or WEBB) will be launched soon to study the atmosphere of these planets. ${ }^{40,41}$ The European Space Agency (ESA) will launch ARIEL (Atmospheric Remote-sensing Infrared Exoplanet Large-survey) to study the chemical composition and thermal structure of transiting planets. ${ }^{42-44}$

MA. In these models, what sort of equations are you using? There are several elements involved in the formation of a planet. We don't understand all the details but we have a good global picture of it. The first element, on which most of the people would agree, is that planets form by an accretion mechanism. ${ }^{45}$ In astrophysics, usually you don't really have this mechanism. Typically, you have a collapse when you have something like a big cloud of gas. The cloud collapses by self-gravity and forms a star. ${ }^{46}$

Planets do not form in this way. They form inside out by gluing together small pieces, which collide, and glue further. When they become massive enough they start accreting material. When they become really massive, they accrete everything around them, typically gas.

This is what happens when a giant planet forms. The core builds up, it becomes a planetesimal, which accretes gas until it becomes a planet. In order to accrete material a planet needs to be within something that feeds it. We call this a protoplanetary disk. ${ }^{47}$ We see them. ${ }^{48}$ We have plenty of examples right now. The disc has also self-gravity. Therefore, there is an interaction between the planet and the disk. Practically the planet steers gravity waves into the disc and the disc reacts with a certain response time, a bit of a lag. This means that the disc itself will induce a gravitational effect on the planet and they're not exactly balanced. This delay produces a tidal force and a torque, which affects the angular momentum of the planet causing the planet to move in all directions depending on the material around. If there are other planets around this planet, the gravitational effects dominate. Moreover if multiple planets are in orbital resonance (orbital periods related by a ratio of small integers) then these effects will be enhanced. ${ }^{49}$

The interaction with the disc wasn't well understood early on. Some people predicted this for the solar system, for the formations of the satellites of the giant planet. However it was never thought to be an important factor for the formation of a planet. Right now these aspects are being considered seriously into the modelling of planetary systems formation.

So these are really the basic equations. If you really want to go into the details of accretion mechanisms, there's a long list of theory, but there isn't a well-defined theory explaining this.

As well as if you migrate the planet, how do you stop it? ${ }^{50}$ When a planet starts migrating it shouldn't stop. If the planet keeps going and if its angular momentum reduces, it will collide into its star. Therefore there has to be a way to hold the planet in a certain position. One mechanism is the decrease in the torque of a planet. ${ }^{51,52}$ Another way involves tidal effects between the star and the planet when they come very close. ${ }^{53}$ There are also magnetic field effects. ${ }^{54}$ So, there are lots of elements that come together, but nobody has a clear picture due to the difficulty of performing measurements of planetary systems during their formation. But we're working on that.

There are bigger telescopes being built, which can see sharper, deeper, and in more detail. One of them is ALMA (Atacama Large Millimetre Array). ${ }^{55,56}$ It consists of an array of millimetric radio telescopes, which can be combined together.

Therefore the theory of the formation of planetary systems is a challenge for the community. But that's one of the key focus of most programs that direct the efforts of the community right now.

\section{References}

1 M. Mayor \& D. Queloz. A Jupiter-mass companion to a solar-type star. Nature 378, 355-359, (1995).

2 G. A. H. Walker, A. R. Walker, A. W. Irwin, A. M. Larson, S. L. S. Yang \& D. C. Richardson. A Search for Jupiter-Mass Companions to Nearby Stars. Icarus 116, 359-375, (1995).

3 W. D. Cochran \& A. P. Hatzes. A high-precision radial-velocity survey for other planetary systems. Astrophysics and Space Science 212, 281-291, (1994).

4 G. W. Marcy \& R. P. Butler. Precision radial velocities with an iodine absorption cell. Publications of the Astronomical Society of the Pacific 104, 270, (1992).

$5 \quad$ R. S. McMillan, T. L. Moore, M. L. Perry \& P. H. Smith. Long, accurate time series measurements of radial velocities of solar-type stars. Astrophysics and Space Science 212, 271-280, (1994).

6 A. P. Boss. Proximity of Jupiter-Like Planets to Low-Mass Stars. Science 267, 360, (1995).

7 R. P. Butler \& G. W. Marcy. A Planet Orbiting 47 Ursae Majoris. The Astrophysical Journal 464, L153-L156, (1996).

8 G. W. Marcy \& R. P. Butler. A Planetary Companion to 70 Virginis. The Astrophysical Journal 464, L147-L151, (1996).

9 R. P. Butler, G. W. Marcy, E. Williams, H. Hauser \& P. Shirts. Three New "51 Pegasi-Type” Planets. The Astrophysical Journal 474, L115-L118, (1997).

10 W. D. Cochran, A. P. Hatzes, R. P. Butler \& G. W. Marcy. The Discovery of a Planetary Companion to 16 Cygni B. The Astrophysical Journal 483, 457-463, (1997).

11 G. W. Marcy, R. P. Butler, S. S. Vogt, D. Fischer \& J. J. Lissauer. A Planetary Companion to a Nearby M4 Dwarf, Gliese 876. The Astrophysical Journal 505, L147-L149, (1998).

12 D. Charbonneau, T. M. Brown, D. W. Latham \& M. Mayor. Detection of Planetary Transits Across a Sun-like Star. The Astrophysical Journal 529, L45-L48, (2000).

13 T. Mazeh, D. Naef, G. Torres, D. W. Latham, M. Mayor, J.-L. Beuzit, T. M. Brown, L. Buchhave, M. Burnet, B. W. Carney, D. Charbonneau, G. A. Drukier, J. B. Laird, F. Pepe, C. Perrier, D. Queloz, N. C. Santos, J.-P. Sivan, S. Udry \& S. Zucker. The Spectroscopic Orbit of the Planetary Companion Transiting HD 209458. The Astrophysical Journal 532, L55-L58, (2000).

14 Kevin A. Bundy \& Geoffrey W. Marcy. A Search for Transit Effects in Spectra 
of 51 Pegasi and HD 209458. Publications of the Astronomical Society of the Pacific 112, 1421-1425, (2000).

15 J. J. Lissauer. Three planets for Upsilon Andromedae. Nature 398, 659-659, (1999).

16 E. J. Rivera, J. J. Lissauer, R. P. Butler, G. W. Marcy, S. S. Vogt, D. A. Fischer, T. M. Brown, G. Laughlin \& G. W. Henry. A 7.5M $\oplus$ Planet Orbiting the Nearby Star, GJ 876. The Astrophysical Journal 634, 625-640, (2005).

17 R. Barnes, B. Jackson, S. N. Raymond, A. A. West \& R. Greenberg. The HD 40307 planetary system: super-Earths or mini-Neptunes? The Astrophysical Journal 695, 1006-1011, (2009).

18 M. Woolfson. The origin and evolution of the solar system. Astronomy \& Geophysics 41, 1.12-11.19, (2000).

19 A. Bouvier \& M. Wadhwa. The age of the Solar System redefined by the oldest $\mathrm{Pb}-\mathrm{Pb}$ age of a meteoritic inclusion. Nature Geoscience 3, 637-641, (2010).

20 C. C. Porco, R. A. West, A. McEwen, A. D. Del Genio, A. P. Ingersoll, P. Thomas, S. Squyres, L. Dones, C. D. Murray, T. V. Johnson, J. A. Burns, A. Brahic, G. Neukum, J. Veverka, J. M. Barbara, T. Denk, M. Evans, J. J. Ferrier, P. Geissler, P. Helfenstein et al. Cassini Imaging of Jupiter\&\#039;s Atmosphere, Satellites, and Rings. Science 299, 1541, (2003).

21 D. N. C. Lin, P. Bodenheimer \& D. C. Richardson. Orbital migration of the planetary companion of 51 Pegasi to its present location. Nature 380, 606-607, (1996).

22 D. E. Trilling, W. Benz, T. Guillot, J. I. Lunine, W. B. Hubbard \& A. Burrows Orbital Evolution and Migration of Giant Planets: Modeling Extrasolar Planets. The Astrophysical Journal 500, 428-439, (1998).

23 P. Goldreich \& S. Tremaine. Disk-satellite interactions. The Astrophysical Journal 241, 425, (1980).

24 C. Baruteau \& J. C. B. Papaloizou. Disk-planets interactions and the diversity of period ratios inkepler's multi-planetary systems. The Astrophysical Journal 778, 7, (2013).

25 J. E. Chambers, G. W. Wetherill \& A. P. Boss. The Stability of Multi-Planet Systems. Icarus 119, 261-268, (1996).

26 H. Rein, J. C. B. Papaloizou \& W. Kley. The dynamical origin of the multi-planetary system HD 45364. A\&A 510, A4, (2010).

27 S. Seager \& D. Deming. Exoplanet Atmospheres. Annual Review of Astronomy and Astrophysics 48, 631-672, (2010).

28 T. P. Greene, M. R. Line, C. Montero, J. J. Fortney, J. Lustig-Yaeger \& K. Luther Characterizing Transiting Exoplanet Atmospheres with JWST. The Astrophysical Journal 817, 17, (2016).

29 I. J. M. Crossfield. Observations of Exoplanet Atmospheres. Publications of the Astronomical Society of the Pacific 127, 941-960, (2015).

30 H. F. Levison, K. A. Kretke \& M. J. Duncan. Growing the gas-giant planets by the gradual accumulation of pebbles. Nature 524, 322-324, (2015).

31 S. Inaba, G. W. Wetherill \& M. Ikoma. Formation of gas giant planets: core accretion models with fragmentation and planetary envelope. Icarus 166, 4662, (2003).

32 A. P. Boss. Rapid Formation of Gas Giant Planets around M Dwarf Stars. The Astrophysical Journal 643, 501-508, (2006).

33 M. Podolak \& S. Zucker. A note on the snow line in protostellar accretion disks. Meteoritics \& Planetary Science 39, 1859-1868, (2004).

34 R. G. Martin \& M. Livio. On the evolution of the snow line in protoplanetary discs. Monthly Notices of the Royal Astronomical Society: Letters 425, L6-L9, (2012).

35 G. D’Angelo \& M. Podolak. Capture and evolution of planetesimals in circumjovian disks. The Astrophysical Journal 806, 203, (2015).

36 MIT. TESS, https://tess.mit.edu/, (2020).

37 G. Ricker, J. Winn, R. Vanderspek, D. Latham, G. Bakos, J. Bean, Z. Berta-Thompson, T. Brown, L. Buchhave, N. Butler, R. P. Butler, W. Chaplin, D. Charbonneau, J. Christensen-Dalsgaard, M. Clampin, D. Deming, J. Doty, N. De Lee, C. Dressing, E. Dunham et al. Transiting Exoplanet Survey Satellite. Journal of Astronomical Telescopes, Instruments, and Systems 1, 014003 , (2014).

38 W. Benz, D. Ehrenreich \& K. Isaak. CHEOPS: CHaracterizing ExOPlanets Satellite. Handbook of Exoplanets, 1257-1281, (2018).

39 ESA. Cheops overview, https://www.esa.int/Science Exploration/Space Science/Cheops/Cheops overview2, (2020).
40 J. P. Gardner, J. C. Mather, M. Clampin, et al. The James Webb Space Telescope. Space Science Reviews 123, 485-606, (2006).

41 NASA. James Webb Space Telescope, https://www.jwst.nasa.gov/, (2020).

42 ESA. Ariel Space Mission, https://arielmission.space/, (2020).

43 G. Tinetti, P. Drossart, P. Eccleston, P. Hartogh, A. Heske, J. Leconte, G. Micela, M. Ollivier, G. Pilbratt, L. Puig, D. Turrini, B. Vandenbussche, P. Wolkenberg, E. Pascale, J.-P. Beaulieu \& M. Güdel. The science of ARIEL (Atmospheric Remote-sensing Infrared Exoplanet Large-survey). Space Telescopes and Instrumentation 2016: Optical, Infrared, and Millimeter Wave 9904, 99041X, (2016).

44 G. Tinetti, P. Drossart, P. Eccleston, P. Hartogh, A. Heske, J. Leconte, G. Micela, M. Ollivier, G. Pilbratt, L. Puig, D. Turrini, B. Vandenbussche, P. Wolkenberg, J.-P. Beaulieu, L. A. Buchave, M. Ferus, M. Griffin, M. Guedel, K. Justtanont, P.-O. Lagage et al. A chemical survey of exoplanets with ARIEL. Experimental Astronomy 46, 135-209, (2018).

45 J. B. Pollack, O. Hubickyj, P. Bodenheimer, J. J. Lissauer, M. Podolak \& Y. Greenzweig. Formation of the Giant Planets by Concurrent Accretion of Solids and Gas. Icarus 124, 62-85, (1996).

46 A. P. Boss. Giant Planet Formation by Gravitational Instability. Science 276, 1836, (1997).

47 J. E. Pringle. Accretion Discs in Astrophysics. Annual Review of Astronomy and Astrophysics 19, 137-160, (1981).

48 M. Keppler, M. Benisty, A. Müller, et al. Discovery of a planetary-mass companion within the gap of the transition disk around PDS 70. A®A 617, A44, (2018).

49 K. Batygin \& A. Morbidelli. Dissipative divergence of resonant orbits. The Astronomical Journal 145, 1, (2012).

50 A. L. Uribe, H. Klahr, M. Flock \& T. Henning. Three-dimensional magnetohydrodynamic simulations of planet migration in turbulent stratified disks. The Astrophysical Journal 736, 85, (2011).

51 F. S. Masset, G. D’Angelo \& W. Kley. On the Migration of Protogiant Solid Cores. The Astrophysical Journal 652, 730-745, (2006).

52 F. S. Masset, A. Morbidelli, A. Crida \& J. Ferreira. Disk Surface Density Transitions as Protoplanet Traps. The Astrophysical Journal 642, 478-487, (2006).

53 D. N. C. Lin \& J. Papaloizou. On the Tidal Interaction between Protoplanets and the Protoplanetary Disk. III. Orbital Migration of Protoplanets. The Astrophysical Journal 309, 846, (1986).

54 M. Wardle. Magnetic fields in protoplanetary disks. Astrophysics and Space Science 311, 35-45, (2007).

55 Z. C. Long, E. Akiyama, M. Sitko, R. B. Fernandes, K. Assani, C. A. Grady, M. Cure, W. C. Danchi, R. Dong, M. Fukagawa, Y. Hasegawa, J. Hashimoto, T. Henning, S.-I. Inutsuka, S. Kraus, J. Kwon, C. M. Lisse, H. B. Liu, S. Mayama, T. Muto et al. Differences in the Gas and Dust Distribution in the Transitional Disk of a Sun-like Young Star, PDS 70. The Astrophysical Journal 858, 112, (2018).

56 ESO. ALMA, https://www.eso.org/public/unitedkingdom/teles-instr/alma/, (2020).

\section{Conflicts of Interest}

There are no conflicts to declare.

\section{Acknowledgements}

M.A. acknowledges support from the President of the UAE's Distinguished Student Scholarship Program (DSS), granted by the UAE's Ministry of Presidential Affairs. SciVPro thanks Dr Subhajit Sarkar for reviewing this manuscript.

\section{Author Contributions}

D.Q. was interviewed by M.A. M.A. wrote the manuscript.

\section{Keywords}

Exoplanets, Migration, Accretion, 51 Pegasi.

\section{Article Information}

This article is licensed by Queloz et al. under a Creative Commons Attribution 4.0 International License (CC BY 4.0). 